\title{
La Fuerza de Tareas Preventivas de los Estados Unidos reafirma su recomendación de rastreo de hipertensión arterial: sintesis de las recomendaciones
}

The US Preventive Services Task Force reaffirms its statements of high blood pressure screening: summary of the statement

Siu AL, US Preventive Services Task Force. Ann Intern Med. 2015;163(10):778-86.

\section{Contexto}

La hipertensión arterial es una condición prevalente que afectaba al $29,1 \%$ de los adultos en los EE. UU. en el año 2011. Su prevalencia aumenta con la edad, siendo del 7,3\% entre los 18 y 39 años, del $32,4 \%$ entre los 40 y 59 años, y del $65 \%$ en los mayores de 60 años. Las personas con hipertensión arterial tienen un riesgo incrementado de mortalidad por eventos cardiovasculares y no presentan ni signos ni síntomas de esta condición, sin embargo una vez diagnosticados son plausibles de un tratamiento adecuado.

En esta publicación, la Fuerza de Tareas de Servicios Preventivos de los Estados Unidos (sigla en inglés, USPSTF) actualiza y reafirma su recomendación de rastreo de hipertensión arterial en adultos mayores de 18 años.

Las ediciones anteriores de esta misma guía (2003 y 2007), ya habían encontrado evidencia de buena calidad para recomendar los beneficios de la toma de presión arterial (PA) como método de rastreo, el cual se asocia a su vez con pocos efectos adversos. Sin embargo éstas no hicieron hincapié en los distintos protocolos de medición.

\section{Métodos de rastreo}

Se consideraron tres métodos diagnósticos de hipertensión arterial: 1) la toma de presión en el consultorio, 2) el monitoreo ambulatorio de presión arterial (MAPA) y 3) el monitoreo domiciliario de presión arterial (MDPA).

La toma de PA en el consultorio es el método más comúnmente utilizado. La mayoría de los estudios clínicos que utilizan esta modalidad registran dos lecturas de PA, en pacientes sentados, luego de cinco minutos de haber entrado al consultorio, usando un manguito de tamaño apropiado y con el brazo del paciente ubicado a la altura de su aurícula derecha. Las tomas múltiples mejoran el valor predictivo positivo con respecto a una toma aislada. La PA se afecta en el corto plazo por múltiples factores, como emociones, estrés, dolor, actividad física, cafeína, nicotina, otras drogas, etc. La presencia del médico es también un factor conocido; entre el 15 y el $30 \%$ de las personas con hipertensión arterial en el consultorio pueden tener cifras de PA más bajas fuera del mismo.

EI MAPA se realiza con dispositivos pequeños y portátiles que registran la PA a intervalos regulares (20 a 30 minutos) durante 12 a 24 horas, mientras que los pacientes realizan sus actividades habituales y también mientras están durmiendo.
EI MDPA se realiza utilizando también dispositivos oscilométricos automatizados y calibrados, pero a diferencia del anterior estos deben ser manipulados por cada paciente (que debe colocarlos, accionarlos y retirarlos) varias veces al día, durante varios días (por lo general se recomienda a los pacientes que realicen dos lecturas de PA separadas por un minuto, dos veces al día, durante cuatro días consecutivos).

La fuerza de tareas encontró evidencia convincente para decir que el MAPA es el mejor método diagnóstico para la hipertensión arterial. Por ello lo recomienda como el estándar de referencia para la confirmación diagnóstica.

Aun así reconoce que el uso del MAPA puede ser problemático en algunas circunstancias y considera aceptable el uso del MDPA cuando se utilizan protocolos apropiados de medición y cuando el MAPA no se encuentra disponible.

\section{Intervalo del control}

El control de la presión arterial en los mayores de 40 años o aquellos individuos con mayor riesgo de hipertensión arterial debería ser anual. Las personas en mayor riesgo son aquellas con presión arterial normal-alta (130 a 139 / 85 a 89 mmHg), aquellos con sobrepeso $\mathrm{u}$ obesidad y los afroamericanos.

Los adultos con PA normal que no tengan otros factores de riesgo deberían controlarse cada tres a cinco años. El control en cualquier caso se hace con la medición de consultorio y la eventual confirmación con un MAPA.

\section{Otras consideraciones}

El rastreo de hipertensión arterial en adultos sin hipertensión conocida puede iniciarse en el consultorio siguiendo la metodología previamente definida. Sin embargo, la fuerza de tareas recomienda su confirmación fuera del consultorio utilizando el MAPA o MDPA.

La mayoría de la evidencia apoya al MAPA como el mejor método para la confirmación diagnóstica de la hipertensión y reconoce una carencia de estudios suficiente sobre el MDPA, sus protocolos de implementación y su comparación con el MAPA. Otras metodologías de control de la PA fuera del consultorio también deben ser evaluadas (puestos de control, enfermería a domicilio, aplicaciones para celulares, control en farmacias, etc.). Las principales recomendaciones de la USPSTF se resumen en la tabla 1.

Tabla 1. Resumen de las recomendaciones

\begin{tabular}{l|l}
\hline \multicolumn{1}{l|}{ Población } & Adultos $\geq 18$ años de edad sin hipertensión conocida. \\
\hline Recomendación & Controlar la presión arterial en busca de hipertensión, y obtener mediciones fuera del consultorio para realizar la confirmación diagnóstica (Grado A). \\
\hline Evaluación del riesgo & $\begin{array}{l}\text { Las personas con mayor riesgo de padecer hipertensión arterial son aquellas con presión arterial normal alta (130 a 139/85 a } 89 \text { mmHg.), aquellas } \\
\text { con sobrepeso u obesidad y los afroamericanos. }\end{array}$ \\
\hline Pruebas de rastreo & $\begin{array}{l}\text { La medición de la presión arterial en el consultorio se realiza con un esfigmomanómetro manual o automático. El protocolo adecuado es usar el } \\
\text { promedio de dos mediciones tomadas con el paciente sentado y habiendo dejado pasar al menos cinco minutos entre la entrada al consultorio y la } \\
\text { medición, usando un manguito de tamaño adecuado y posicionando el brazo del paciente a la altura de su aurícula derecha. Las mediciones } \\
\text { múltiples a lo largo del tiempo tienen un mejor valor predictivo positivo que una medición aislada. } \\
\text { El monitoreo ambulatorio o el domiciliario de la presión arterial puede ser usado para la confirmación diagnóstica de la hipertensión arterial luego del } \\
\text { rastreo inicial. }\end{array}$ \\
\hline Intervalo entre controles de rastreo & $\begin{array}{l}\text { Los adultos } \geq 40 \text { años de edad y las personas con riesgo aumentado para hipertensión arterial deben ser controlados anualmente. Los adultos entre } \\
18 \text { y } 39 \text { años de edad con presión arterial normal (<130/85 mmHg.) y que no tienen otros factores de riesgo deben ser controlados cada tres a cinco } \\
\text { años. }\end{array}$ \\
\hline Balance entre beneficios y perjuicios & El beneficio neto del control de la presión arterial en adultos es substancial.
\end{tabular}

Balance entre beneficios y perjuicios $\quad$ El beneficio neto del control de la presión arterial en adultos es substancial. 


\section{Recomendaciones de otras sociedades}

El Joint National Committee VIII en 2014 no hace referencia al diagnóstico de la hipertensión arterial. El Joint National Committee VII recomendaba el control de la PA cada dos años en adultos con cifras menores a $120 / 80 \mathrm{mmHg}$ y anualmente para aquellos con cifras entre 120 a 139 / 80 a 89 mmHg. La American Heart Association recomienda el control en cada visita médica 0 al menos cada dos años para adultos con PA menor a 120/80 mmHg. La American Academy of Family Physicians hace recomendaciones similares a las de la USPSTF y el American Congress of Obstetricians and Gynecologists recomienda el control de la presión en las mujeres como parte de la visita ginecológica anual.

\section{Comentario}

Aun cuando el abordaje de la hipertensión arterial comprende otros aspectos (prevención, tratamiento) al profundizado en esta guía (diagnóstico), tanto en la esfera individual como para la salud pública, es enormemente valioso rescatar la importancia del gesto inicial de la toma de PA en el consultorio en una persona que se acerca sin síntomas. Este rol central determina la posibilidad más substancial del viraje en la historia natural de esta condición. Este es el valor más importante de esta guía y sobre el que habrá que volver periódicamente.

Según hemos podido observar, este documento coloca al MAPA en el lugar del estudio más adecuado; aunque su falta de disponibilidad universal obliga a los autores a ampliar su recomendación al MDPA y dejar también abierta la posibilidad al uso de otros métodos de tomas repetidas de PA fuera del consultorio.

Jorge J. Janson [ Servicio de Clinica Médica del Hospital Italiano de Buenos Aires jorge.janson@ @ospitalitaliano.org.ar ]

Janson JJ. La Fuerza de Tareas Preventivas de los Estados Unidos reafirma su recomendación de rastreo de hipertensión arterial: síntesis de las recomendaciones. Evid Act Pract Ambul 2016;19(2):64-65. Abr-Jun. Comentado de: Siu AL. U.S. Preventive Services Task Force. Screening for high blood pressure in adults: U.S. Preventive Services Task Force recommendation statement. Ann Intern Med. 2015 Nov 17;163(10):778-86. PMID: 26458123

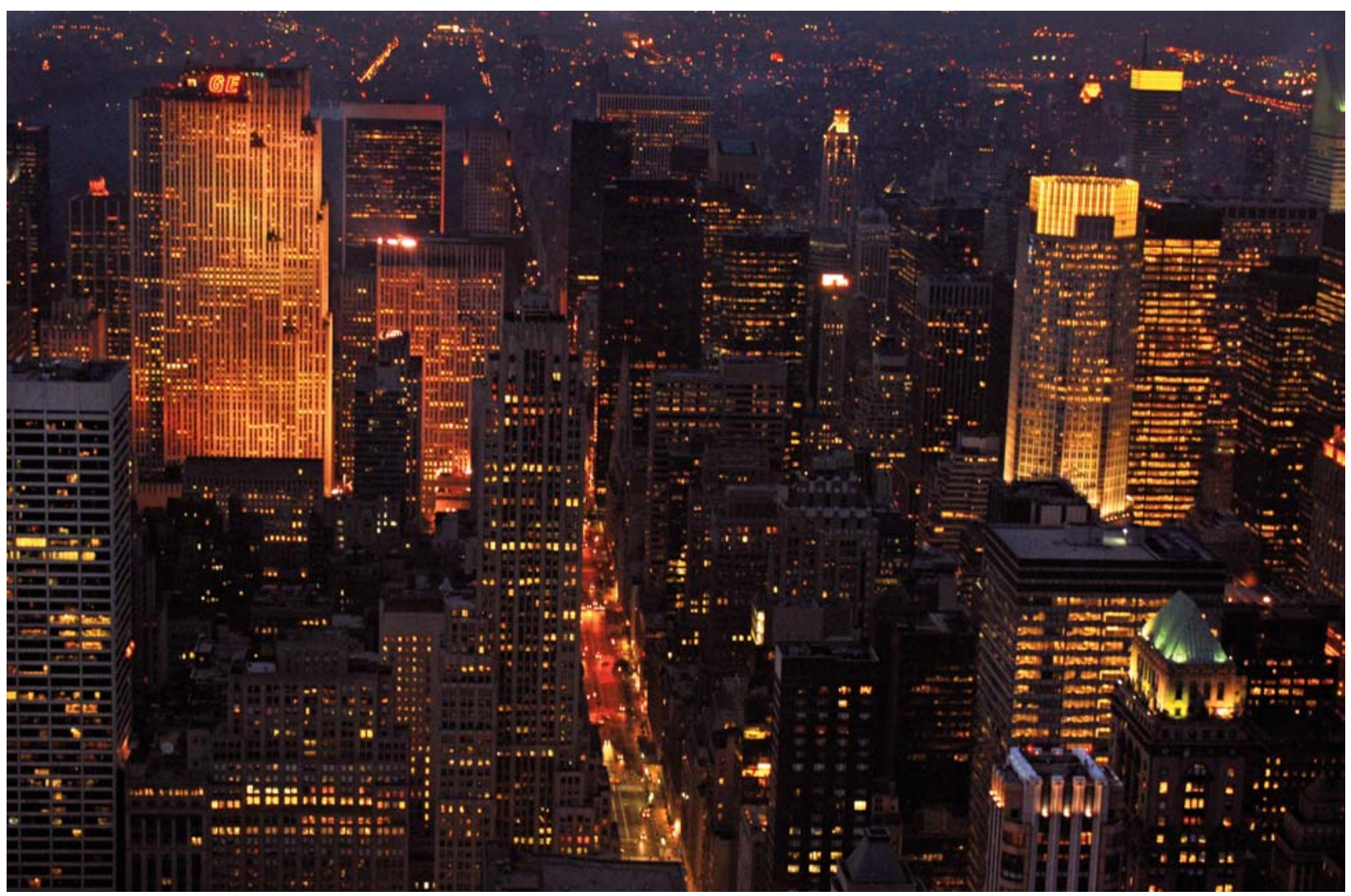

Cortesía: Silvina Spina 